\section{FUNCTIONALLY UNIQUE SYSTEM}

DMP is proud to announce DMPRAM: 'Dental Maintenance Plan Recruitment, Assessment and Monitoring', the new software application developed for patient assessment, recruitment and monitoring of payment plan processing.

The system allows you to observe and assess your patients' current oral and dental condition to establish the appropriate grade and fee for a plan that suits both you and your patient. It lets you monitor the fee grade versus related income. As well as these benefits DMPRAM allows you to carry out even more admin on your computer, making your practice more efficient and greener too.

DMPRAM is linked to the DMP secure web portal which offers access to the practice's payment plans database as well as many documents which can be downloaded and printed off for patient enrolment and information. You can easily download your latest monthly financial report on plan patient activity, together with the reports from the previous eight months on this 24/7 portal. There are sections showing each dentist's current grades and fees and full patient lists with their enrolment details. This functionally unique system is used through a secure route ensuring absolute privacy for you and your patients.

For further information call 08000699687 or visit www. dentalmaintenanceplan.co.uk.

\section{PRACTICE SALES WITH NO REGRETS}

If you are contemplating selling your practice within the next 12 months, and you know that the term of your lease has only a few years to run, Chris Pomfret from rhw solicitors in Guildford recommends contacting your landlord without delay. Find out if your landlord is amenable to a lease extension now and endeavour to agree terms. You will not regret this when you come to sell the practice.

rhw solicitors LLP is a Surreybased law firm providing specialist legal advice on a wide range of legal matters. They have specialist knowledge in commercial matters; in particular they are renowned for dealing with healthcare providers. They also offer a strong private client team of solicitors to meet the individual's needs.

Chris Pomfret heads the specialist dental team at rhw solicitors and has extensive expertise in dealing with the sale and purchase of dental practices and all the surrounding legal issues. He can be contacted by phone on 01483540546 or by email atchris.pomfret@rhw.co.uk.

\title{
SCRAPER TARGETS TONGUE BACTERIA
}

Created by Dr Robert Wagstaff in 2000, Orabrush is a patented, FDA-approved tongue cleaner designed to help cure bad breath using a combination of ultra-soft, pointed bristles that reach

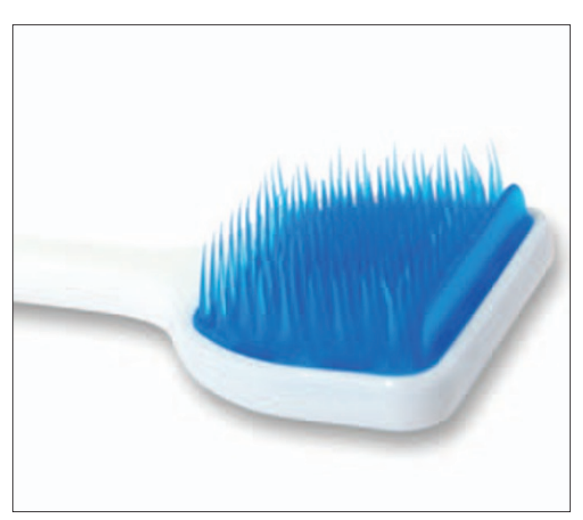

deep into the tongue and a unique scraper to remove bacteria.

The ultra-soft, pointed bristles reach deep into the uneven crevices of your tongue and loosen bacteria. These bristles are designed after the surgeon's scrub brush, the same brush they use to clean bacteria from their hands before beginning a procedure. This differs from toothbrush bristles, which are designed for the smooth surfaces of your teeth, not your tongue. The scraper then collects and removes the bacteria and residue generated from brushing, helping cure bad breath $90 \%$ of which is caused by bacteria on the tongue.

\section{HIGHLY ACCURATE CARIES DETECTION}

The CarieScan PRO not only indicates when restoration of a tooth is required, but also specifically locates the caries lesion, allowing restorations to be as small and cosmetically acceptable as possible.

Patients and dentists benefit from a much quicker and more comfortable experience as a result of a functionality upgrade which increases the speed of measurements. Each measurement now takes less than a second, down from the five seconds of the previous version. Additionally the classification reading of the $0-100$ scale has also been further simplified into High, Medium and Low $(\mathrm{H}, \mathrm{M}, \mathrm{L})$ probability categories, supported by advice specific to each identified category, supporting the dentist with caries treatment and intervention support.

The PRO enables proactive dental care and an easy way to continuously monitor dental health, allowing easy monitoring of the effect of treatment regimes to show decay advancement or regression, thus enabling dentists to tailor treatment to the patients' needs.
The PRO is $94.8 \%$ accurate in detecting caries and healthy tooth structure. Using AC Impedance Spectroscopy technique, it recognises decay much earlier. The CarieScan PRO registers seven times fewer false positives than its closest competitor, minimising the potential to drill on healthy teeth.

www.cariescan.com 